\title{
Constructing customized modelling guidelines: a Participatory Integrated Assessment and Modelling example
}

\author{
$\underline{\text { Fateme Zare }}^{\text {a }}{ }^{(D)}$, Joseph H.A. Guillaume ${ }^{a}$ and Anthony J. Jakeman $^{\text {a }}$ \\ ${ }^{a}$ Fenner School of Environment and Society, Australian National University, Building 141, Linnaeus \\ Way, ACT, Canberra 2601, Australia, \\ Email: Fateme.zare@anu.edu.au
}

\begin{abstract}
As scientific modelling and integrated assessment have matured, a number of best practice guidelines have emerged. While standard guidelines play an important role in setting expectations for repetitive modelling tasks, many problems require a flexible, adaptive approach, meaning that analysts cannot simply follow a single guideline, but rather have to draw on several to construct a process to address their problem. New combined guidelines or meta-guidelines often add further complexity rather than solving the issue. In this paper, we describe two simple ideas to combine multiple guidelines in a way that provides a customizable modelling process: "pathway diagrams" and "ID cards". The former involves thinking of an analysis process in terms of a unique "pathway", divided into a set of steps, including decision forks and points for reflection and iteration. This pathway provides a structure within which steps from guidelines can be inserted. The second idea involves identifying steps recommended within guidelines and annotated them to link them to an "ID card" for that step. Each ID card then provides a quick summary of what has been said about a step across multiple guidelines. While these ID cards can pull in information from a variety of sources, they remain an individual/group's personal summary of the state of knowledge about a step, providing a common language and point of interaction for thinking about and discussing what steps can be inserted into a pathway and why. We provide an example pathway, its steps and an example of the ID card. Specifically, we build and demonstrate a Participatory Integrated Assessment and Modelling pathway drawing on key, highly cited articles. It is hoped that the pathway diagram will help implement the idea of modelling as an adaptive spiral process, containing several iterative loops to reach a fit for purpose model and agreement between stakeholders.
\end{abstract}

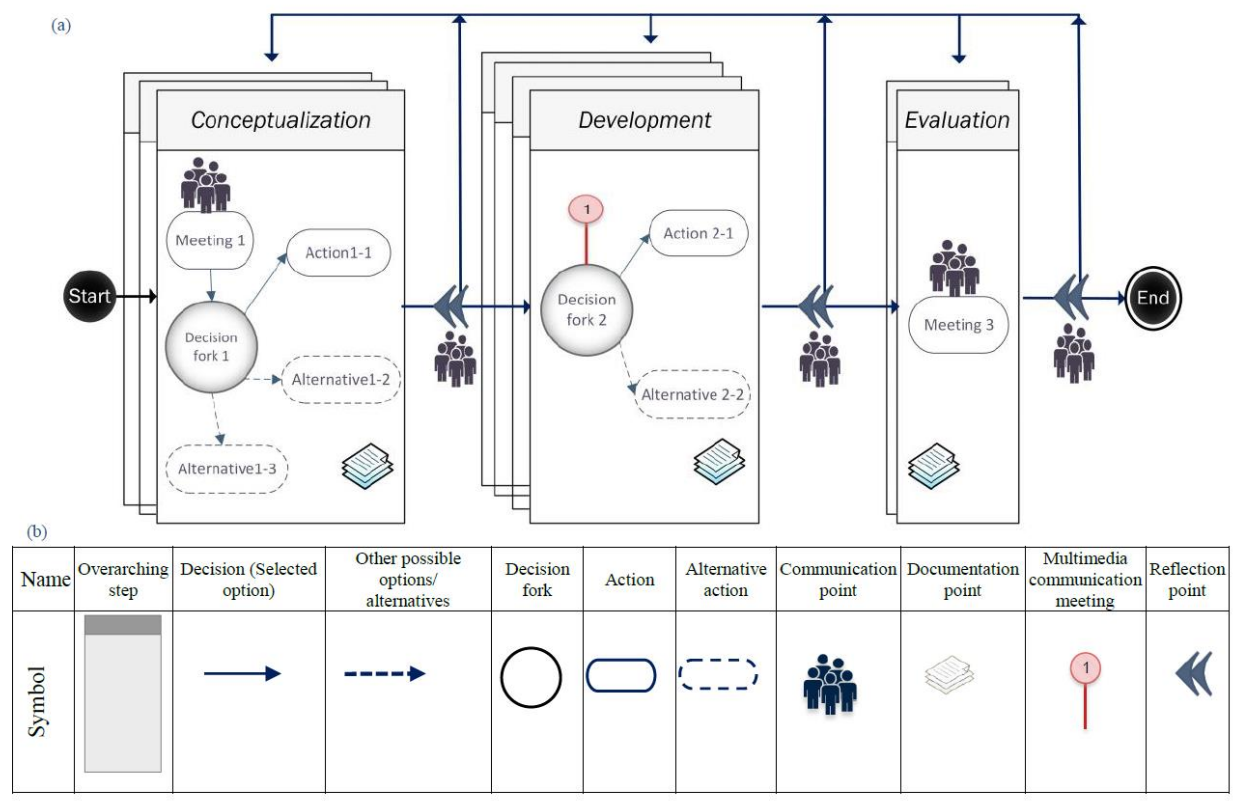

Figure 1. A pathway example (a) and legend (b), demonstrating the use of the pathway diagram components (Zare et al., 2019)

Keywords: Integrated modelling, participatory modelling, Pathway, ID cards 
Zare et al, Constructing customized modelling guidelines: a Participatory Integrated Assessment and Modelling example

\section{INTRODUCTION}

The popularity of integrated assessment and modelling (IAM) has been increasing over the last 40 years (Zare et al., 2017). IAM has passed through conception, formation and practice phases, and is now in a maturity phase where uncertainty management, stakeholder involvement, and assessing economic and social aspects require more investigation (Voinov and Bousquet, 2010; Zare et al., 2017). In the practice phase, IAM frameworks, methodologies, approaches and principles were defined and tested, resulting in several best practice guidelines of great value for guiding repetitive modelling tasks (e.g. Badham et al.,(2019) for Integrated Water Resource Management). However, nowadays, complex problems require a flexible, adaptive approach, in which analysts need to use several guidelines and standards to address their problem. New combined instructions or meta-guidelines often add further complexity and therefore, they do not necessarily solve the issue.

As a particular example, IAM research has a strong focus on biophysical modelling, (Zare et al., 2017), reflected in many modelling guidelines and best practices mainly focusing on the model as a tool to assess biophysical issues. Socio-economic aspects have received less attention, but stakeholder involvement has become almost a "must" (Voinov and Bousquet, 2010), with its distinctive literature. As a result, it makes sense to bring these threads together.

One of the most cited articles articulating the integrated modelling steps from a modeller's perspective (864 citations based on Google Scholar) is "Ten iterative steps in development and evaluation of environmental models" (Jakeman et al., 2006). It describes ten steps for model development as part of minimum standards for model development and reporting. The steps describe the modelling process, starting from problem formulation and ending with a model to pass to the end-user. While the article emphasizes the importance of stakeholder engagement in this process, it does not explicitly show how and when to involve them, focusing more on the quantitative part of the modelling process. Badham et al., (2019) published a complementary follow-up paper which lays out a series of questions that the modeller should ask when undertaking a modelling exercise.

On the other hand, there is much research which shows how to do participatory modelling from a social scientist point of view. One highly cited article (913 citations based on Google Scholar) is "Modeling with stakeholders" (Voinov and Bousquet, 2010). This article goes into more depth into how and when to involve stakeholders in the modelling process and reviews different terms in modelling with stakeholders. Among those terms, we use the more generic "participatory modelling".

In addition to the need to combine guidelines, transparent information about project progress is a challenge. There is a need for a common way (tool/language) to speak and communicate among stakeholders, to help everyone reach a common understanding of steps and requirements, and to improve knowledge sharing.

This article bridges these two streams of guidelines, the more quantitative, 'natural science' approach to modelling and the more qualitative, 'social' approach. We used "pathway diagrams" and "ID cards" as tools to provide a common language and flexible framework to achieve a coherent description of a modelling process, explicitly showing the steps involved, and decisions and points at which stakeholders are involved. The result of this paper is a "Participatory Integrated Assessment Modelling" pathway (PIAM) based on this literature (Jakeman et al., 2006; Voinov et al., 2018; Voinov and Bousquet, 2010) and an example of the type of ID card to be associated with each step of the PIAM, summarizing essential information.

\section{METHOD}

\subsection{Pathways}

Integrated assessment and modelling (Jakeman and Letcher, 2003), like any other decision support process, involves a sequence of steps including decision forks, choices, points for reflection and iteration which can be described as a unique "pathway" (Lahtinen et al., 2017). The concept of the pathway is still emerging in modelling research, and it has not been used as a basis for integrating steps. A pathway, however, provides a structure within which steps from guidelines can be inserted. There are many decisions involved in each step, and each decision fork might have many options. In order to achieve customized model guidelines, a practitioner can select the essential steps, decisions and alternatives relevant to their situation, as we will demonstrate for an integrated participatory modelling project.

A modelling pathway is dependent on the context and circumstances of the project, and might need several iterations, or change in the order of the steps, to meet the project goals and achieve an adaptive and agile process. Iterations usually happen after each reflective communication point. In each reflective communication point, the outcome of that step is presented to stakeholders and project team members to obtain their feedback. 
Zare et al, Constructing customized modelling guidelines: a Participatory Integrated Assessment and Modelling example

Reflective evaluation is acquired at this point to determine the next steps in the path, which might involve another iteration or continuing on to the next step. Validation is critical to confirm or debunk the results of an iteration (Voinov and Bousquet, 2010).

Figure 1 shows a pathway example to illustrate how a pathway can graphically capture and document an integrated participatory modelling project in terms of steps and decisions at each step (Zare et al., 2019). The legend table in Figure 1 shows the main elements of a pathway which are the steps, decision forks, actions, documentation and reflection points. For a full description of the essential elements of a pathway diagram, please refer to Zare et al. (2019).

This specific pathway example consists of three main steps. In the first step, labelled as a "conceptualization" step, a meeting has occurred where a decision is made between three different alternative options and Action 1-1 is selected. There is also a documentation point which contains information about the step, decisions and alternative options, people who were involved and the results. There is a reflective communication point to reflect on the conceptualization step and its results. If the stakeholders and modellers do not agree on the result, an iteration is needed. This time the decision will be made based on knowledge gained in the previous iteration, so it is a new layer in this step. Graphically, we showed the iterations as three layers for the step. In the next step, "development", the decision fork has two options and to select between them a multimedia communication meeting has occurred, documented in a report. This report and the development step enabled reflection which ended up with iteration through four different rounds. In the last step, the final meeting and reflection on the final report occur. Even the evaluation step may not go as planned, such that a reflection meeting involving stakeholders is necessary to reflect on the last step and the pathway overall. In this diagram, this results in another iteration of the evaluation step.

Overall, the project pathway can be described as an adaptive spiral process (Figure 2) which benefits from insights acquired during regular reflective communication meetings and becomes more mature overtime to meet stakeholder needs. We should not force the project into a predefined protocol or procedure and should be adaptive to changes and go in circles and branch out, be prepared to go back, reiterate and refine (Voinov and Bousquet, 2010). Having regular reflective communication helps to improve the efficiency and effectiveness of the project (Zare et al., 2019) during these iterations.



\subsection{ID cards}

Figure 2. Modelling as an adaptive spiral process

The application of ID cards in the modelling pathway can be compared to the use of archetypes in System Thinking. Archetypes describe dynamic phenomena and "common stories" that repeatedly occur in the diverse sets of behaviour and contexts in the system. Braun (2002) presents summarized information about ten of the most well-known archetypes using a consistent structure. Each archetype description consists of the name and general description, dynamic theory, behaviour over time, application, example, prescriptive actions, action steps and rationale. We used this idea to introduce Steps ID Cards. We acknowledge there are several closely related ideas, including "good practices", "recipes" and "design patterns".

The idea of the "ID card" focuses on collecting what is known about each step as an organized collection of explicit statements. Steps' ID Cards are a collection of statements which show categorized information about the necessary steps of the modelling process and can be used as a common story to provide a better understanding of the process between modellers and stakeholders. Each ID card provides a quick summary of what has been said about a step across multiple guidelines. As described in the introduction, in this case, the ID cards are based on selected articles in the field (Jakeman et al., 2006; Voinov et al., 2018; Voinov and Bousquet, 2010), but they can be modified and completed by adding more detail and reviewing other research. ID cards remain an individual/group's customized summary of the state of knowledge about a step, providing a common language and point of interaction for thinking about and discussing what steps can be inserted into a pathway and why. The intention is to give a formalized description of key steps of interest, including specifications such as their aim, involved actors and main decisions, without attempting the impossible task of gathering a comprehensive pathway in the modelling process. More precisely, we accept that uncertainty is inevitable in describing a step, in terms of both accuracy and completeness of the information included. An ID 
Zare et al, Constructing customized modelling guidelines: a Participatory Integrated Assessment and Modelling example

card is therefore expected to always be open to modifications, with the content being progressively refined over time.

Steps recommended within each guideline document (i.e. Jakeman et al., 2006; Voinov et al., 2018; Voinov and Bousquet, 2010) are identified and annotated to link them to their respective ID card. Using the information in the articles, we extracted and categorized the essential information that practitioners need to know about each step. The categories used here were: the Name of step; Objective, which speaks about aim and purpose; Rationale, which contains information about why/where/when/in which situation and how we need this step; Decisions/tasks during the step; Tools which are available to assist reaching the objectives; and Actors who should be involved. We filled out one set of cards for each of the selected guidelines. As each card consists of a collection of statements, the ID cards from multiple articles are combined by merging the corresponding statements, paying attention to ordering and merging/rewording of duplicate statements. This characteristic of ID cards theoretically enables practitioners to pull in information from a variety of sources. As noted in the introduction, we used two highly cited papers in integrated modelling (Jakeman et al., 2006) and participatory modelling (Voinov and Bousquet, 2010) which describe the steps of a modelling project from two different points of view, along with a comprehensive review on the tools and models of participatory modelling (Voinov et al., 2018).

\section{RESULTS AND IMPLICATIONS}

Figure 3 shows three different visualizations of the modelling pathway in terms of steps. We developed the participatory integrated modelling pathway based on elements of different stages of a participatory modelling process (Voinov et al., 2018) and ten iterative modelling steps (Jakeman et al., 2006). The developed pathway shows the essential steps to suit the practitioners' needs. These steps are aligned and linked to ID cards (e.g. "Conceptualization", see Figure 4). Some steps in the original articles have been merged together as they are not the current focus of attention (e.g. "Model Evaluation") and some points have been added in, e.g. "reflective evaluation points". Reflective evaluation points occur after each step where all the actors decide if the objectives of the step (which are mentioned in the associated ID card) are satisfied or if there is a need to iterate and repeat the step.

Similar to the first two pathways, the participatory integrated modelling pathway is intended as a generic one summarizing essential steps and in practice could be changed based on context. The steps in Figure 3 can be shuffled, iterative and adapted based on project context and situation. Each of the pathway steps in a real project could be divided into more detailed ones, and also could be repeated as many times as needed to reach an agreement between all actors on the result of the step. Together with ID cards, the pathway can play the role of a guideline to provide a common view of a project among participants, improving knowledge sharing.

Figure 3 demonstrates how views of pathways from multiples sources can be reorganized and merged into a new one to suit practitioners' needs based on the modelling context. The pathway of a modelling project is commonly described as a chain of iterative steps at which various decisions are made. The pathway diagram can be a convenient tool, helping to use different resources easily and develop a pathway that suits our aim. Given the importance of improving the knowledge sharing between all actors in a participatory modelling or planning exercise, the pathway diagram provides an accessible tool to understand and communicate over steps and process. Modellers might know which step they are working on and what is the next step by experience, but one needs to share this knowledge and engender a shared understanding of the pathway. Also, not everyone in an integrated modelling team has the same description of each step; here is where the ID card idea can help to reach a shared understanding.

Each of the steps in Figure 3 is associated with an ID card, which provides sufficient information to understand the detail of the step. Figure 4 presents the conceptualization step's ID card as an example. The conceptualization step has an important role in communication with the stakeholders, and among the modelling team members. The reviewed literature covers this step in some detail, which is why it was selected as an example. This ID card illustrates the objective and rationale of this step, who are the main actors involved, what decisions the actors need to make, what the main tasks are and what tools are available to achieve them. The information on this ID card is extracted from multiple sources (Jakeman et al., 2006; Voinov et al., 2018; Voinov and Bousquet, 2010) and merged at a statement-level after grouping into sections. 
Zare et al, Constructing customized modelling guidelines: a Participatory Integrated Assessment and Modelling example

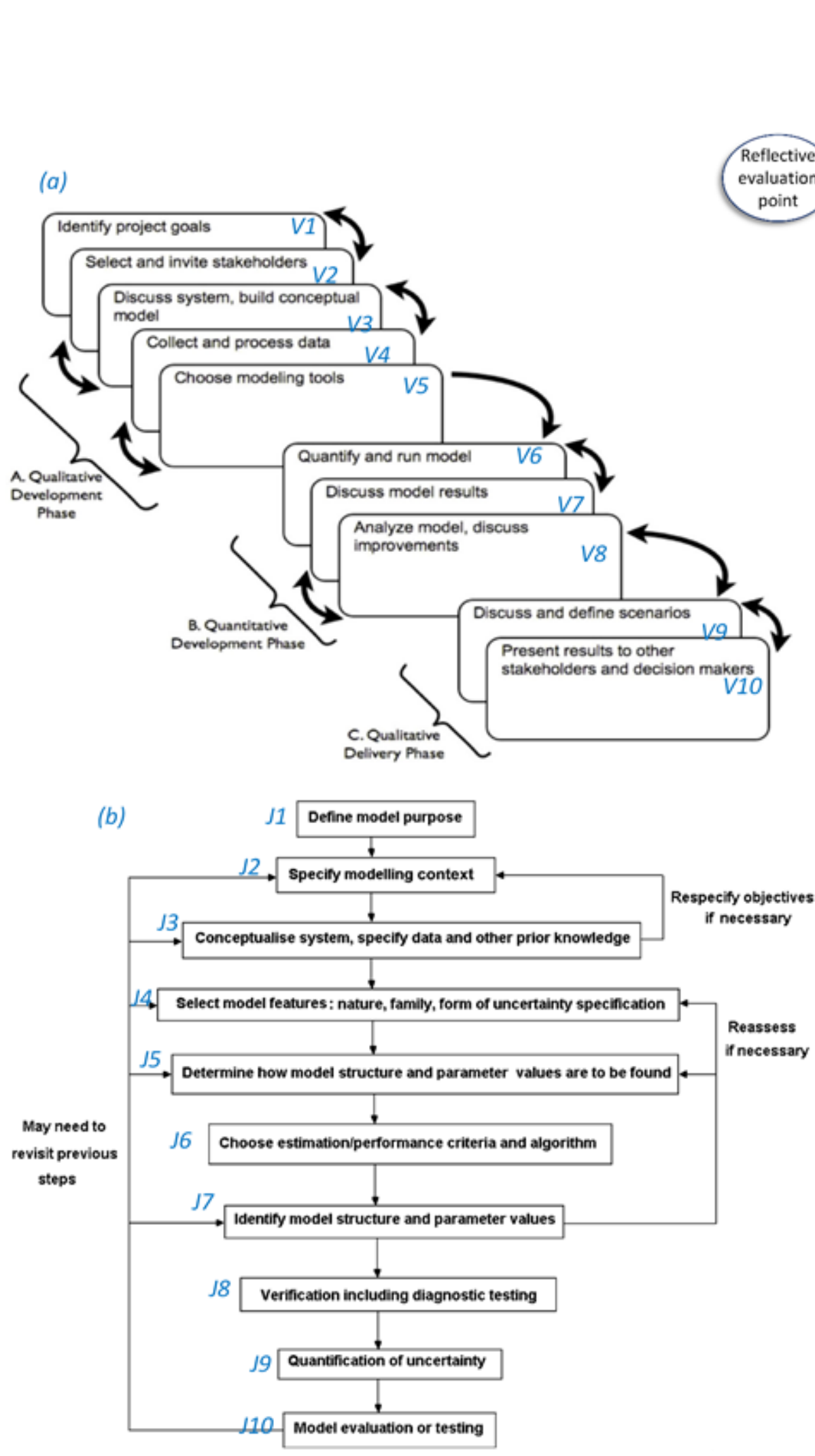

Figure 3. Three visualisations of pathways in terms of steps. (a) Different stages of a participatory modelling process (Voinov et al., 2018), (b) Ten iterative modelling steps (Jakeman et al., 2006), (c) Participatory integrated modelling pathway, combining and rewording elements of (a) and (b) to suit the practitioner's needs, allowing linking to ID cards (e.g. "Conceptualization", see Figure 4), and merging steps that are not the current focus of attention (e.g. "Model Evaluation"). This demonstrates how views of pathways from multiple sources can be reorganized and merged

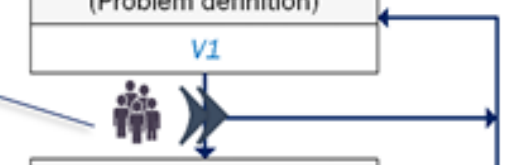

Identify stakeholders

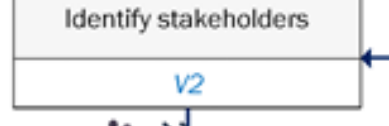

部)
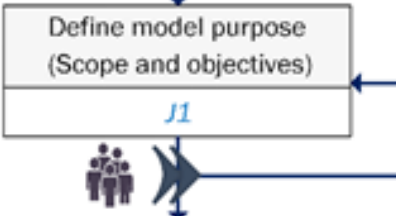

Specify modelling context

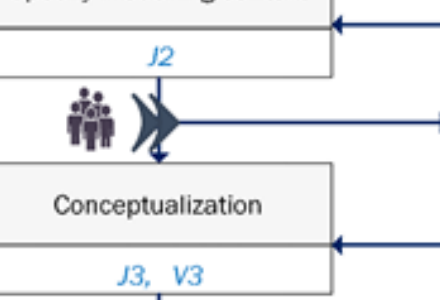

คำ $)$

Model specification, data and knowledge collection $J 3, J 4, V 4, V 5$

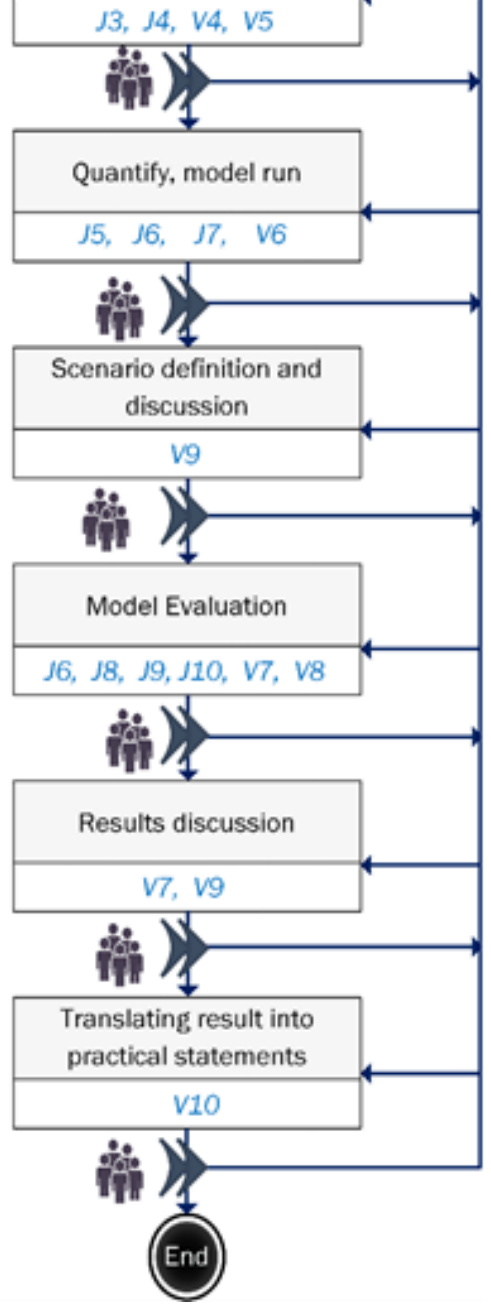


Zare et al, Constructing customized modelling guidelines: a Participatory Integrated Assessment and Modelling example

\section{ID card name: Conceptualization of the system \\ Objective}

- Project participants build conceptual, visual representations of the components of the problem being considered.

- Conceptualisation refers to basic premises about the working of the system being modelled.

- Consideration and justification of options in defining the system warrant attention by modellers

Rationale and their clients.

- The conceptualisation step is important even if a model is not designed from scratch because time and money (as well as the clients' beliefs) restrict one to using a 'canned' model.

Conceptualisation exposes the weaknesses of the canned approach and perhaps ways to mitigate them.

\section{Decisions/tasks}

- $\quad$ It is always helpful to start building a conceptual model with stakeholders.

- What to include and what not to incorporate in a modelling activity should be addressed explicitly at the outset and iteratively revisited as far as resources allow, consider an iterative process to allow and encourage modifications, and changes to the model.

- The conceptualisation may be rudimentary, with details postponed until the results of knowledge elicitation and data analysis can be exploited.

- A tentative initial conceptualisation and a visualisation such as a block diagram may be a great help in showing what else must be found out about the system.

- The system being modelled should be defined clearly, including its boundaries (e.g. physical, socioeconomic and institutional).

- Boundary conditions can then be modelled as constraints or as input scenarios, whose values can be perturbed in line with stipulated assumptions.

- identifying, articulating, and representing the relationships among the many components of a problem; on the spatial, temporal relationships; on how changes in one area affect other factors that may be important to solutions and to stakeholder concerns

Tools

Use aids to thinking to show how model drivers are linked to internal (state) variables and outputs (observed responses), e.g. an influence diagram, linguistic model, block diagram, bond graph, Rich pictures (RP), Cognitive/concept mapping (CCM), Causal loop diagram (CLD), Cultural consensus (CC), Decision tree analyses (DTA)

- $\quad$ Methods for Semi-quantitative modelling (conceptual quantification): Fuzzy cognitive mapping (FCM), Scenario building (SB), Social network analysis (SNA), Analytic hierarchy process (AHP)

- $\quad$ Mind mapping exercises, participatory workshops with boxes and arrows, engaging stakeholders in role- playing games all these are good tools to initiate the process. This method corresponds better to the genuine co-construction approach

- Propose a preliminary model, created previously by the scientists. Stakeholders can then propose modifications and start questioning the draft model. This approach is often less time consuming than starting from scratch.

- In any case, it is important to consider an iterative process to allow and encourage modifications, and changes to the model.

Actors Scientist (modellers and their clients), officials, other interested parties

Figure 4. Conceptualization step ID card. Demonstrates how information from multiple sources can be synthesized at a statement-level after grouping into sections. Green statements are from (Jakeman et al., 2006) and blue statements are from (Voinov et al., 2018; Voinov and Bousquet, 2010)

There is substantial literature on the modelling process, best practices and manuals and tool kits, but a real modelling process is always dependent on the context. Therefore, practitioners might use different resources to figure out how to cover their needs in different contexts. An ID card acts as a venue for the practitioner to pull together information to describe what a step means to participants involved in the pathway. The ID cards are helpful as a way of summarizing and merging because it is common for information from different resources to be complementary. When reviewing different resources to develop the set of ID cards (which are linked to Figure 3), information is merged based on the objective of the step rather than name, because researchers with a different background might use slightly different terms for a specific step, but still, mostly agree about objectives. We structured the ID card in a way to suit our purpose, and other practitioners can modify the structure to include and emphasize the specific information they need. 
Zare et al, Constructing customized modelling guidelines: a Participatory Integrated Assessment and Modelling example

Objectives and actors of the conceptualization step (Figure 4) were mostly similar in the sources used, with slightly different views and wording. Objectives were emphasized more in Jakeman et al. (2006) and tools are described more in Voinov et al. (2018). There is still information missing. For example, the rationale only covers a narrow case and could be broadened by using more literature. Also, decisions and tasks are not specified and do not go into detail, so as it stands, the ID card might not be sufficiently useful as a to-do list, but still it gives information about the main tasks. This simply reflects the type of information included in the resources used. In this ID card for the conceptualization step (Figure 4), we aimed to stay as close as possible to the original wording. Clearly, it is possible to write some parts more concisely and remove some details which might not be needed for the practitioner.

\section{CONCLUSION}

There is a need to go beyond strict guidelines to provide adaptivity in the modelling process to be able to confront complex issues and engage different stakeholders. We suggest new formats to share information about steps, to combine information flexibly from multiple guidelines to make a shared, common source of information. We introduced the participatory integrated modelling pathway and its set of ID cards, which practitioners can use to share or develop their own version of the pathway and its steps, customized to the context at hand while still providing a common language between actors. Pathway diagram and ID cards are useful tools to develop common knowledge and to facilitate communication between stakeholders and modellers, and modellers and other disciplines in order to reach a shared understanding of the modelling process and its steps. Developing pathway and ID cards is an ongoing process of building a shared vision and understanding. However, one should not use them to force stakeholders into a predefined protocol and process (Voinov and Bousquet, 2010). On the contrary, this is intended as a tool to motivate and facilitate an agile and adaptive participatory integrated modelling process.

It is hoped that the pathway diagram will help implement the idea of modelling as an adaptive spiral process, with several iterative loops to reach a fit for purpose model and agreement between stakeholders. Future research could scale up and improve the participatory integrated modelling pathway (Figure 3, c) and the ID cards by reviewing more resources, maturing presentation and providing user-friendly tools.

\section{REFERENCES}

Badham, J., Elsawah, S., Guillaume, J.H.A., Hamilton, S.H., Hunt, R.J., Jakeman, A.J., Pierce, S.A., Snow, V.O., Babbar-Sebens, M., Fu, B., Gober, P., Hill, M.C., Iwanaga, T., Loucks, D.P., Merritt, W.S., Peckham, S.D., Richmond, A.K., Zare, F., Ames, D., Bammer, G., 2019. Effective modeling for Integrated Water Resource Management: A guide to contextual practices by phases and steps and future opportunities. Environ. Model. Softw. 116, 40-56. https://doi.org/10.1016/j.envsoft.2019.02.013

Braun, W., 2002. The system archetypes. System 2002, 27.

Jakeman, A.J., Letcher, R.A., 2003. Integrated assessment and modelling: Features, principles and examples for catchment management. Environ. Model. Softw. 18, 491-501. https://doi.org/10.1016/S13648152(03)00024-0

Jakeman, A.J., Letcher, R.A., Norton, J.P., 2006. Ten iterative steps in development and evaluation of environmental models. Environ. Model. Softw. 21, 602-614. https://doi.org/10.1016/j.envsoft.2006.01.004

Lahtinen, T.J., Guillaume, J.H.A., Hämäläinen, R.P., 2017. Why pay attention to paths in the practice of environmental modelling? Environ. Model. Softw. 92, 74-81. https://doi.org/10.1016/j.envsoft.2017.02.019

Voinov, A., Bousquet, F., 2010. Modelling with stakeholders. Environ. Model. Softw. 25, 1268-1281. https://doi.org/10.1016/j.envsoft.2010.03.007

Voinov, A., Jenni, K., Gray, S., Kolagani, N., Glynn, P.D., Bommel, P., Prell, C., Zellner, M., Paolisso, M., Jordan, R., Sterling, E., Schmitt Olabisi, L., Giabbanelli, P.J., Sun, Z., Le Page, C., Elsawah, S., BenDor, T.K., Hubacek, K., Laursen, B.K., Jetter, A., Basco-Carrera, L., Singer, A., Young, L., Brunacini, J., Smajgl, A., 2018. Tools and methods in participatory modeling: Selecting the right tool for the job. Environ. Model. Softw. 109, 232-255. https://doi.org/10.1016/j.envsoft.2018.08.028

Zare, F., Elsawah, S., Iwanaga, T., Jakeman, A.J., Pierce, S.A., 2017. Integrated water assessment and modelling: A bibliometric analysis of trends in the water resource sector. J. Hydrol. 552, 765-778. https://doi.org/10.1016/j.jhydrol.2017.07.031

Zare, F., Guillaume, J.H.A., Jakeman, A.J., Torabi, O., 2019. Reflective communication can improve project pathways: an integrated environmental modelling case study-Under review. Environ. Model. Softw. under revi, $1-34$. 